\title{
Kinder als ,Stakeholder“ in Kindertageseinrichtungen [KiSte] - Studie zu den Sichtweisen der Kinder auf institutionelle Arrangements
}

\author{
Tom Witton • Katja Gramelt • Agata Skalska • Werner Thole
}

Online publiziert: 21. April 2020

(C) Der/die Autor(en) 2020

Zusammenfassung Das Forschungsprojekt Kinder als „Stakeholder“ in Kindertageseinrichtungen (KiSte) zielt auf die empirisch fundierte Erfassung der unterschiedlichen Sichtweisen von Kindern auf ihre Kindertageseinrichtung (Kita). Es wird davon ausgegangen, dass Kinder ihre eigenständigen Ideen zur Ausgestaltung der frühpädagogischen Arrangements haben, diese aber nicht durchgängig im Diskurs um die Weiterentwicklung frühpädagogischer Arrangements wahrgenommen werden. Da Kinder neben den Pädagog*innen die zentralen Akteur*innen und Gestalter*innen des pädagogischen und sozialen Alltags in Kitas sind, erhalten sie im Forschungsvorhaben die Möglichkeit, für sie Relevantes und Zeigenswertes zu filmen und in parallel verlaufenden Interviews ihre jeweiligen Deutungsweisen darzulegen.

Schlüsselwörter Kindertageseinrichtungen · Pädagogik der Kindheit · Ethnographie · Videographie

\footnotetext{
T. Witton, M.A. $(\varangle) \cdot$ Prof. Dr. W. Thole Institut für Sozialwesen, Fachbereich 01 - Humanwissenschaften, Universität Kassel, Arnold-Bode-Straße 10, 34127 Kassel, Deutschland

E-Mail: tom.witton@uni-kassel.de
}

Prof. Dr. W. Thole

E-Mail: wthole@uni-kassel.de

Prof. Dr. K. Gramelt · A. Skalska, M.A.

Institut für Sozial- und Kulturwissenschaften, Hochschule Düsseldorf,

Münsterstr. 156, 40476 Düsseldorf, Deutschland

Prof. Dr. K. Gramelt

E-Mail: katja.gramelt@hs-duesseldorf.de

A. Skalska, M.A.

E-Mail: agata.skalska@hs-duesseldorf.de 


\title{
Children as "Stakeholders" in Daycare facilities [KiSte] - Perspectives of Children on their daily and institutionalized arrangements
}

\begin{abstract}
The research project Children as "Stakeholders" in Daycare facilities is the empirically based assessment of the different perspectives of children on their day care center. It is assumed that children have their own ideas for the design of early childhood education and care arrangements, however these are not consistently perceived in the discourse of early childhood education and care arrangements. Since children are the central actors and designers of daily pedagogical and social life alongside the pedagogues, the research project gives them the opportunity to film relevant aspects and to describe their perspectives in parallel interviews.
\end{abstract}

Keywords Daycare facilities for children · Pedagogy of childhood · Ethnography · Videography

Das Vorhaben, Kinder als eigenständige wie eigensinnige Akteur*innen wahrzunehmen und zu adressieren und kindlichen Perspektiven eine stärkere Aufmerksamkeit zukommen zu lassen, motiviert seit vier Jahrzehnten zunehmend sichtbarer die Forschung zu Fragestellungen der Kindheit, den Bedingungen kindlichen Aufwachsens und zur Sozialisation von Kindern (vgl. Bühler-Niederberger 2019). Im Diskurs um Kinder und Kindheiten und in den Versuchen, diese Lebensphase angemessen theoretisch zu beschreiben, kommt der Frage nach der Rolle der Kinder als Kinder (Olk 2009, S. 153) und danach, welche Position ihnen in der jeweiligen Gesellschaft, aber auch in den Forschungsprozessen und -designs zugeschrieben wird, eine besondere Bedeutung zu. Forschungsansätze der Kindheitsforschung versuchen entsprechend, die Rolle des Kindes neu zu denken und zu operationalisieren. Dies dokumentiert sich unter anderem in der Konzipierung von „Kindern als Akteur*innen“ und dem Versuch, so den aktiven Beitrag der jüngsten Gesellschaftsmitglieder und deren Wirkmächtigkeit auf gesellschaftliche Prozesse hervorzuheben (Prout und James 1990, S. 8). Ohne die Verwobenheit von Kindern in gesamtgesellschaftliche Ordnungen, wie beispielweise Generationen, mitzudenken, sind die entsprechenden Fragen nicht zu klären. Nachdrücklich wird seit Jahren darauf hingewiesen, dass spezifische Forschungszugänge erforderlich sind, um die Perspektiven von Kindern zu erfassen (vgl. u.a. Heinzel 2013) und dabei die Ambivalenzen, die mit dem Konzept von Kindern als Akteur*innen einhergehen, jedoch nicht zu ignorieren (vgl. Betz und Eßer 2016, S. 302). Die Abkehr von einer erwachsenenzentrierten Perspektive auf soziale und pädagogische Wirklichkeiten hin zu einer, die dem Kind, „das fähig ist, sich eine eigene Meinung zu bilden“ das Recht zusichert, „diese Meinung in allen das Kind berührenden Angelegenheiten frei zu äußern“" (UN-Kinderrechtskonventionen, Art. 12, Abs. 1), erfordert die Entwicklung von Forschungszugängen, die Kindern ermöglichen, ihre Welt- und Selbstdeutungen zu artikulieren. Der im Titel des hier vorgestellten Forschungsprojekts Kinder als „Stakeholder “ in Kindertageseinrichtungen (KiSte) enthaltene Begriff „Stakeholder“ soll den sich darüber ergebenden forschungsmethodologischen Ansprüchen entsprechen und das Verhältnis der Kinder zu ihrer Einrichtung als mit eigenständigem Interesse an der Gestaltung 
und Konzipierung der Institution in den Blick rücken. Zugleich sind theoretische, wie empirische Blicke auf Kinder und Kindheiten herausgefordert, zu beobachtende ökonomische wie zweckrationale Vereinnahmungen und Instrumentalisierungen von Kindern und Kindheiten wie auch damit einhergehende gesellschaftliche Optimierungsstrategien kritisch zu reflektieren. Der Anspruch des Forschungsvorhabens KiSte ist entsprechend einerseits, die bislang weitgehend als passiv zu beschreibende Rolle der Kinder im Diskurs um Qualität in Kindertageseinrichtungen und in den Forschungsvorhaben zu thematisieren. Andererseits sind die forschungsmethodologischen Grenzen zu berücksichtigen und die Schwierigkeiten und Herausforderungen der ,einfachen“ Perspektivübernahme mitzudenken.

Ausgehend von diesen Überlegungen zielt das Forschungsvorhaben KiSte darauf $\mathrm{ab}$, dass Kinder in Kindertageseinrichtungen ihre Sichtweisen kommunizieren - konkreter: Erstens wird über das Forschungsvorhaben empirisch fundiertes Wissen bezüglich der Sichtweisen auf und Deutungen von Kindertageseinrichtungen durch Kinder generiert. Vermutet wird, dass Kinder keineswegs in einer identischen Form Kindertageseinrichtungen erleben und die Formen, wie sich Kinder Räume und Artefakte aktiv aneignen und gestalten, ebenso unterschiedlich sind, wie die Weisen, Beziehungen zu gestalten und ihnen eine Relevanz zuzuweisen. Angestrebt wird, eine empirisch valide und theoretisch begründete Typologie der - wahrscheinlich komplexen, nuancenreichen und diffizilen - kindlichen Sichtweisen. Zweitens wird angestrebt, das gewonnene Datenmaterial für die Aus-, Fort- und Weiterbildung von Pädagog*innen - Erzieher*innen und akademisch qualifizierte Pädagog*innen - didaktisch aufzubereiten. In dem zweiten Teilbereich des Forschungsprojekts wird entsprechend dieses Arbeitsvorhabens das erhobene Material unter Bezug auf die Rekonstruktionen des ersten Arbeitsprogramms bearbeitet und Pädagog*innen an Fachschulen und in Studiengängen der Pädagogik der Kindheit vor respektive nach ihrer praktischen Qualifizierungsphase gezeigt und mit ihnen diskutiert. Die kindlichen Perspektiven auf und Deutungen von Kindertageseinrichtungen können so in qualifizierender Intention nähergebracht werden.

Direkt und unmittelbar schließt die geplante Untersuchung an die jüngere Kindheitsforschung an, in welcher von einer kodifizierten Eigenständigkeit von Kindern ausgegangen wird (vgl. u.a. Braches-Chyrek et al. 2011). Im Kontrast zu entwicklungspsychologischen sowie auch klassischen pädagogischen und sozialisationstheoretischen Annahmen werden Kinder als handlungsfähige und teil-autonome Akteur*innen ihrer Sozialwelt verstanden, womit ihren Wahrnehmungen und Interpretationen im Selbst-Welt-Verhältnis Eigenständigkeit und Wirkmächtigkeit zugestanden wird. Kinder sind demnach ,nicht nur in der Lage, hilfreiche Informationen $\mathrm{zu}$ geben und richtig zu interpretieren, sondern verstehen sogar Aufforderungen in eine kooperative Richtung“ (Tomasello 2010, S. 30). Explizit diese Erkenntnis aufgreifend und das Wissen über die Bilder von Kindern der pädagogischen Fachkräfte in Kindertageseinrichtungen beachtend (vgl. Kuhn und Neumann 2015; Oehlmann 2012), wird in dem Forschungsvorhaben davon ausgegangen, dass Kinder früh in der Lage sind, sich selbstständig zu positionieren. Zugleich wird reflektiert, dass die sich darin äußernde Autonomie im Kontext der gegebenen ,generationalen Ordnung“ unter den Kindern in gegebenen zeitlichen, räumlichen und sozialstrukturellen Bedingungen, also im Rahmen der vorliegenden Asymmetrien, 
wie Ungleichheitsdimensionen, zu verstehen und zu analysieren sind (vgl. Sünker und Bühler-Niederberger 2014; Betz 2008) und die Gestaltungen sich ebenfalls nicht unabhängig von Normierungen und Normalisierungen vollziehen können (vgl. u.a. Kelle und Mierendorff 2013).

Im Zentrum der Untersuchung stehen ethnographisch grundierte und von Kindern gestaltete Begehungen von Kindertageseinrichtungen. Kinder zeigen mittels von ihnen selbstgeführten Videokameras den Forscher*innen ihre Kindertageseinrichtung und legen damit ihre Sichtweise auf selbige offen. Die Videokamera dient als distanzschaffendes Medium. Die Kinder machen dabei einen Schritt zurück und begeben sich auf die Metaebene, von der aus sie für einige Momente ihre Einrichtung betrachten. Dies schafft einerseits die Möglichkeit einer analytischen Distanz, andererseits verlängert es die Zeit, die sich die Kinder aktiv kognitiv mit der Situation im Sinne eines ,stepping back“ und ,prolonged involvement“ (Stephenson 2009) beschäftigen. Die Videographien werden von Seiten der Forscher*innen durch dialoggestützte Interviews begleitet, in denen sie sich flexibel auf kindliche Ausdrucksformen einstellen (vgl. Punch 2002). Die Kinder zeigen, filmen und erläutern für sie relevante Orte und Akteur*innen innerhalb der Institution, vorhandene Artefakte, die Infrastruktur sowie pädagogische Praktiken. Die Aufgabe der Forscher*innen ist dabei, den Kindern größtmöglichen Spielraum bei der Auswahl des Gezeigten zu lassen, ihnen die Möglichkeiten zu geben, sich frei zu allen von ihnen als erwähnenswert markierten Sachverhalten, Orten, Personen und Gegenständen zu äußern und während oder nach der Begehung der Einrichtung gezielt Nachfragen zu Erlebtem bzw. Gezeigtem zu stellen. Zur Teilnahme eingeladen werden alle fünf- bis sechsjährigen Kinder der jeweils besuchten Einrichtung.

Die Forscher*innen begeben sich damit auf für sie „fremdes Terrain“ (Friebertshäuser und Panagiotopoulou 2010, S. 304) und erhalten einen Einblick in die Kultur der Kinder, die sich in Abgrenzung zu der von Erwachsenen als „fremde Kultur" beschreiben lässt (Deckert-Peaceman et al. 2010, S. 72) und in der Kinder nicht als werdende Erwachsene, sondern als ",actors in their own rights“ (Borgers et al. 2000, S. 61) anerkannt werden. Das forschungsmethodische Design des skizzierten Vorhabens beruht auf vier forschungspraktischen methodischen Verfahren der Datengewinnung und -auswertung. In einem multimodalen Setting werden ein ethnographischer Zugang, videographische Elemente und Interviews mit Kindern miteinander verbunden und in den methodologischen Ansatz der Grounded Theory (vgl. Glaser und Strauss 1967) eingebettet.

Die Erkundung der „Wirklichkeit des Pädagogischen“ aus Kinderperspektive, von Michael-Sebastian Honig $(1999,2010)$ als ethnographische Kinderforschung bezeichnet, zielt über die reine Darstellung hinaus auch darauf ab, die subjektiven Perspektiven der Kinder auf pädagogische Gestaltungsmerkmale offen zu legen und damit für die Weiterentwicklung institutioneller Bildungseinrichtungen bearbeitbar zu machen. Empirisch auswertbare Daten werden zum einen aus den von den Kindern erstellten Bildfolgen und zum anderen aus den die Erstellung der Bildfolgen begleitenden Beschreibungen und Argumentationen gewonnen. Der ethnographische Zugang findet sich in der teilnehmenden Beobachtung der Forscher*innen, die es ermöglicht, die Perspektive der Kinder im Vergleich zur Erwachsenenperspektive kontrastiv einzuordnen (vgl. Heinzel et al. 2010; Friebertshäuser und Panagiotopou- 
lou 2010). Durch dieses Vorgehen und das damit generierte Material sollen die Ergebnisse sowohl zur Grundlegung und weiteren Ausdifferenzierung des Forschungsstandes zur Bedeutung von Kindertageseinrichtungen im Aufwachsen von Kindern beitragen, als auch relevante Hinweise für eine qualitative Weiterentwicklung und Professionalisierung der institutionellen Bildungs-, Erziehungs- und Betreuungsarrangements liefern, indem Wissen über die subjektiven und eigenwilligen Relevanzsetzungen und Bedürfnisse von Kindern zur Bearbeitung zur Verfügung gestellt wird (vgl. Gramelt 2014, S. 457). Außerdem wird im Laufe der Forschungsarbeit reflektiert, ob und inwieweit sich das Forschungssetting eignet, die kindlichen Sichtweisen zu erfassen, welche forschungsmethodischen Anpassungen erfolgen müssen, um die vielschichtigen Ausdrucksformen von Kindern wahrzunehmen und wie flexibel Forschungssetting und Forscher*innen auf die Konfrontationen mit der für sie „fremden“ und in sich heterogenen Kinderkultur reagieren müssen.

Funding Open Access funding provided by Projekt DEAL.

Open Access Dieser Artikel wird unter der Creative Commons Namensnennung 4.0 International Lizenz veröffentlicht, welche die Nutzung, Vervielfältigung, Bearbeitung, Verbreitung und Wiedergabe in jeglichem Medium und Format erlaubt, sofern Sie den/die ursprünglichen Autor(en) und die Quelle ordnungsgemäß nennen, einen Link zur Creative Commons Lizenz beifügen und angeben, ob Änderungen vorgenommen wurden.

Die in diesem Artikel enthaltenen Bilder und sonstiges Drittmaterial unterliegen ebenfalls der genannten Creative Commons Lizenz, sofern sich aus der Abbildungslegende nichts anderes ergibt. Sofern das betreffende Material nicht unter der genannten Creative Commons Lizenz steht und die betreffende Handlung nicht nach gesetzlichen Vorschriften erlaubt ist, ist für die oben aufgeführten Weiterverwendungen des Materials die Einwilligung des jeweiligen Rechteinhabers einzuholen.

Weitere Details zur Lizenz entnehmen Sie bitte der Lizenzinformation auf http://creativecommons.org/ licenses/by/4.0/deed.de.

\section{Literatur}

Betz, T. (2008). Ungleiche Kindheiten. Theoretische und empirische Analysen zur Sozialberichterstattung über Kinder. Weinheim und Basel: Juventa.

Betz, T., \& Eßer, F. (2016). Kinder als Akteure - forschungsbezogene Implikationen des erfolgreichen Agency-Konzepts. Diskurs Kindheits- und Jugendforschung, 11(3), 301-314.

Borgers, N., De Leeuw, E., \& Hox, J. (2000). Children as respondents in survey research: cognitive development and response quality. Bulletin de Methodologie Sociologique, 6(6), 60-75.

Braches-Chyrek, R., Bühler-Niederberger, D., Heinzel, F., Sünker, H., \& Thole, W. (2011). Deutungen und Bilder von Kindern und Kindheiten. In Promotionskolleg ,Kinder und Kindheiten im Spannungsfeld gesellschaftlicher Modernisierung“ (Hrsg.), Kindheitsbilder und die Akteure generationaler Arrangements (S. 9-18). Wiesbaden: VS.

Bühler-Niederberger, D. (2019). Lebensphase Kindheit. München: Beltz Juventa.

Deckert-Peaceman, H., Dietrich, C., \& Stenger, U. (2010). Einführung in die Kindheitsforschung. Darmstadt: WBG.

Friebershäuser, B., \& Panagiotopoulou, A. (2010). Ethnographische Feldforschung. In B. Friebertshäuser, A. Langer \& A. Prengel (Hrsg.), Handbuch Qualitative Forschungsmethoden in der Erziehungswissenschaft (3. Aufl. S. 301-322). Weinheim und München: Juventa.

Glaser, B. G., \& Strauss, A. L. (1967). The discovery of grounded theory: strategies for qualitative research. Chicago: Aldine.

Gramelt, K. (2014). Diversität in der frühkindlichen Bildung. In R. Braches-Chyrek, C. Röhner, H. Sünker \& M. Hopf (Hrsg.), Handbuch frühe Kindheit (S. 451-459). Opladen: Budrich. 
Heinzel, F. (2013). Zugänge zur kindlichen Perspektive - Methoden der Kindheitsforschung. In B. Friebertshäuser, A. Langer \& A. Prengel (Hrsg.), Handbuch qualitative Forschungsmethoden in der Erziehungswissenschaft (4. Aufl). Weinheim und München: Juventa.

Heinzel, F., Thole, W., Cloos, P., \& Köngeter, S. (2010). „Auf unsicherem Terrain“. Ethnographische Forschung im Kontext des Bildungs- und Sozialwesens. Wiesbaden: VS.

Honig, M.-S. (1999). Entwurf einer Theorie der Kindheit. Berlin: Suhrkamp.

Honig, M.-S. (2010). Beobachtung (früh-)pädagogischer Felder. In G. E. Schäfer \& R. Staege (Hrsg.), Frühkindliche Lernprozesse verstehen. Ethnographische und phänomenologische Beiträge zur Bildungsforschung (S. 91-101). Weinheim und Basel: Juventa.

Kelle, H., \& Mierendorff, J. (Hrsg.). (2013). Normierung und Normalisierung der Kindheit. Weinheim und Basel: Juventa.

Kuhn, M., \& Neumann, S. (2015). Verstehen und Befremden. Objektivierungen des „Anderen“ in der ethnographischen Forschung. Zeitschrift für qualitative Forschung, 16(1), 25-42.

Oehlmann, S. (2012). Kindbilder von pädagogischen Fachkräften: eine Studie zu den Kindbildern von Lehrkräften und Erzieherinnen. Weinheim und Basel: Juventa.

Olk, T. (2009). Ungleichheit und Gerechtigkeit im Generationenverhältnis. Sind Kindheit und Kinder die Verlierer der Sozialstaatsreform? In M.-S. Honig (Hrsg.), Ordnungen der Kindheit. Problemstellungen und Perspektiven der Kindheitsforschung (S. 127-153). Weinheim und München: Belz Juventa.

Prout, A., \& James, A. (1990). A new paradigm for the sociology of childhood? Provenance, promise and problems. In A. James \& A. Prout (Hrsg.), Constructing and reconstructing childhood. Contemporary issues in the sociological study of childhood (S. 7-34). London: Routledge.

Punch, S. (2002). Research with children. The same or different from research with adults? Childhood, 9(3), 321-341.

Stephenson, A. (2009). Horses in the sandpit: photography, prolonged involvement and „stepping back“ as strategies for listening to children's voices. Early Child Development \& Care, 179(2), 131-141.

Sünker, H., \& Bühler-Niederberger, D. (2014). Kindheit und Gesellschaft. In R. Braches-Chyrek (Hrsg.), Handbuch Frühe Kindheit (S. 41-52). Opladen: Budrich.

Tomasello, M. (2010). Warum wir kooperieren. Frankfurt am Main: Suhrkamp. 